Check for updates

Cite this: RSC Adv., 2017, 7, 28951

Received 5th January 2017

Accepted 18th May 2017

DOI: $10.1039 / c 7 r a 00179 g$

rsc.li/rsc-advances

\section{Emulsion-based systems for fabrication of electrospun nanofibers: food, pharmaceutical and biomedical applications}

\author{
Nooshin Nikmaram, ${ }^{a}$ Shahin Roohinejad, (D) $\dagger^{* b c}$ Sara Hashemi, ${ }^{c}$ \\ Mohamed Koubaa, (D) ${ }^{d}$ Francisco J. Barba, (D) Alireza Abbaspourrad ${ }^{f}$ \\ and Ralf Greiner ${ }^{\mathrm{b}}$
}

\begin{abstract}
Electrospinning is considered a promising technology for fabricating ultrafine fibers via the application of electrostatic repulsive forces. Electrospun nanofibers produced via emulsion electrospinning are widely used as delivery systems to encapsulate bioactive compounds and drugs in food and pharmaceuticals, respectively. Emulsion electrospinning has also gained significant interest for the production of vehicles for sustained and controlled release. There are several parameters affecting the properties of fabricated fibers including the type of emulsion, emulsion composition, electric field strength, conductivity of solution, surface tension, electrode configuration, solution cooling time, dissolution temperature, and solution flow rate; therefore, all these parameters should be precisely controlled to obtain optimum results. Some of the advantages of these fibers are the protection of encapsulated materials from environmental conditions, room temperature processes, release rate control and high loading efficiency. This study presents an overview of the emulsion electrospinning method, its mechanism of action and its applications in both the food and pharmaceutical fields.
\end{abstract}

\section{Introduction}

Over the past decade, electrospinning has gained significant interest in both the scientific community and industry (e.g. food and biomedical industries) for ultrafine fiber fabrication. ${ }^{1}$ The electrospinning process is a straightforward, versatile and lowcost technique that employs a high-voltage electrostatic field in the polymer solution or melt, via a metallic capillary orifice, to fabricate ultrathin fibrous scaffolds with fiber diameters ranging from nanometer- to micron-sized. ${ }^{2}$ The produced nanofibers offer notable physicochemical characteristics,

\footnotetext{
${ }^{a}$ Young Researches and Elite Club, Sabzevar Branch, Islamic Azad University, Sabzevar, Iran

${ }^{b}$ Department of Food Technology and Bioprocess Engineering, Max Rubner-Institut, Federal Research Institute of Nutrition and Food, Haid-und-Neu-Straße 9, 76131 Karlsruhe, Germany. E-mail: Shahin.roohinejad@mri.bund.de; Tel: +49 7216625540 'Burn and Wound Healing Research Center, Division of Food and Nutrition, Shiraz University of Medical Sciences, Shiraz, Iran

${ }^{a}$ Sorbonne Universités, Université de Technologie de Compiègne, Laboratoire Transformations Intégrées de la Matière Renouvelable (UTC/ESCOM, EA 4297 TIMR), Centre de Recherche de Royallieu, CS 60319, 60203 Compiègne Cedex, France ${ }^{e}$ Nutrition and Food Science Area, Preventive Medicine and Public Health, Food Science, Toxicology and Forensic Medicine Department Faculty of Pharmacy, Universitat de València, Avda. Vicent Andrés Estellés, s/n, 46100 Burjassot, València, Spain

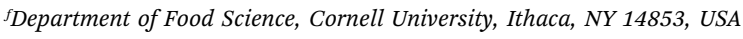

$\dagger$ Alexander von Humboldt postdoctoral research fellow.
}

including a significantly large surface-to-mass ratio, great porosity, and a remarkable mechanical performance. ${ }^{3}$

Generally, ultrafine fibers with controllable and adjustable mechanical properties, porosity and flexibility can be produced by electrospinning a mixture solution of a bioactive compound/ drug, solvent and a polymer. However, some problems have been reported regarding the fabrication of nanofibers using traditional electrospinning. For instance, the application of this system causes severe primary burst release of ingredients and is unable to provide the desired requirements such as the sustained release of bioactive compounds/drugs or perform cell differentiation, which is a challenge in the food and pharmaceutical industries. ${ }^{4}$

Emulsion electrospinning is a new and simple method of electrospinning to produce core-shell nanofibers, which has sparked increasing interest since the process is considered more "stable". ${ }^{5}$ Many researchers have developed electrospinning techniques based on using emulsion systems to incorporate functional materials (e.g. food bioactive compounds, enzymes, proteins, drugs, etc.) into biodegradable polymer fibers to form core-shell structures. ${ }^{6,7}$ Compared to the traditional electrospinning techniques, the application of the emulsion electrospinning method is a promising alternative as it allows the encapsulation of lipophilic compounds using low-cost hydrophilic polymers and avoids the use of organic solvents, which are highly restricted in food systems. ${ }^{8}$ Moreover, the application of this system has been reported to result in the sustained release, good bioactivity and effectiveness of encapsulated drugs after delivery and release, and 


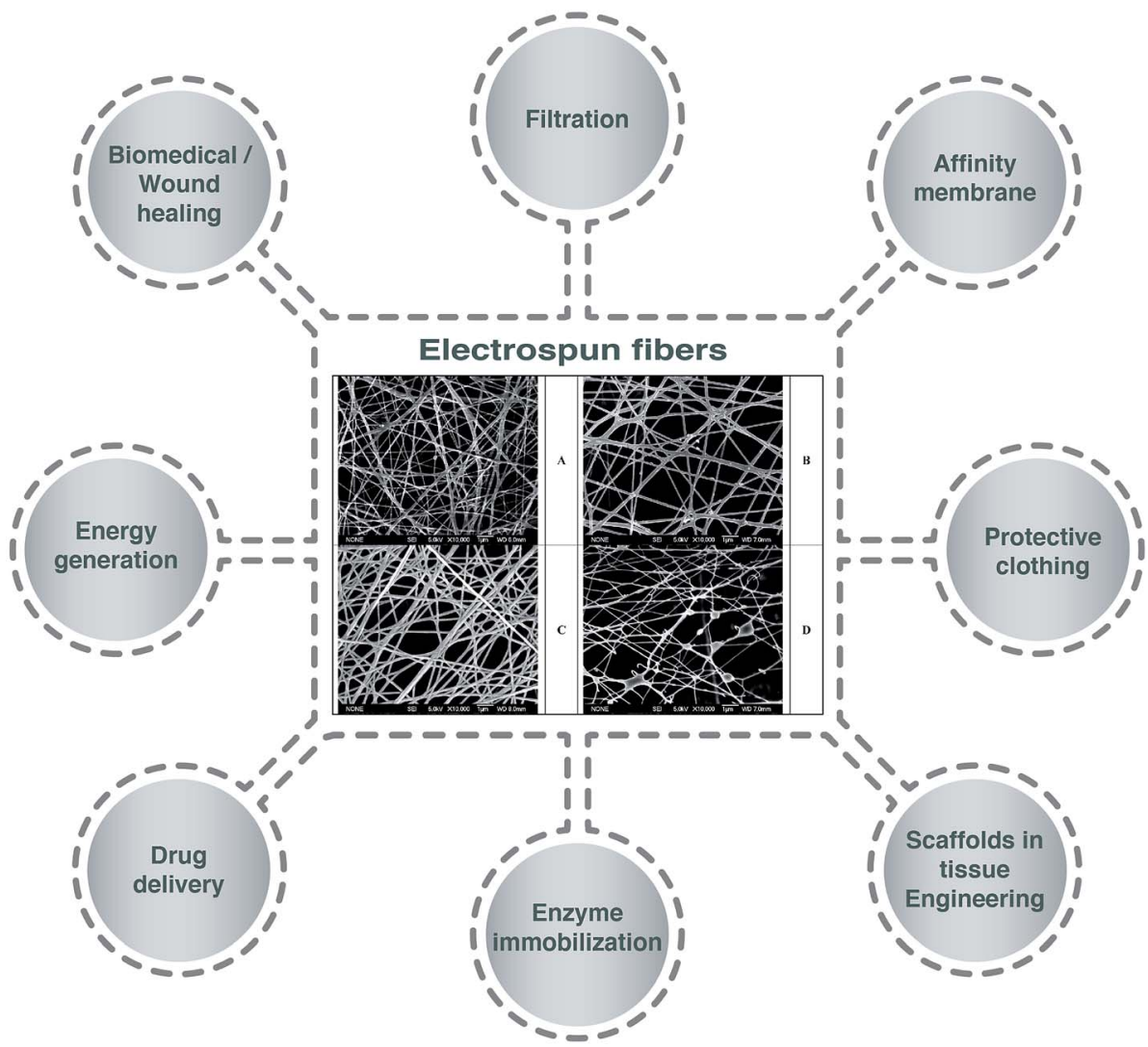

Fig. 1 Schematic displays of the application of electrospun fibers in different sectors. Scanning electron micrographs of electrospun 7.5 wt\% poly(vinyl alcohol) solution containing (A) 10 wt\% Surfynol 465, (B) 10 wt\% Surfynol 465 loaded with 1.5 wt\% eugenol, (C) 7.5 wt\% Surfynol 465 loaded with 1.125 wt\% eugenol, (D) 10 wt\% Surfynol 465 loaded with 1.125 wt\% eugenol. ${ }^{3}$

to simplify the metabolism, proliferation, and differentiation of cells. ${ }^{4}$ Fig. 1 represents applications of electrospun fibers in different sectors with selected morphologies of electrospun fibers in the center.

When emulsion electrospinning is used to produce nanofibers, there are some factors that could affect the fiber properties including (i) the type of emulsion, (ii) electric field strength, (iii) solution conductivity, (iv) surface tension, (v) electrode configuration, (vi) solution cooling time, (vii) dissolution temperature and (viii) solution flow rate. ${ }^{9}$ Different electrospinning parameters and polymer solutions may lead to the production of different morphologies; thus, precise control of operating conditions and solution parameters are required to obtain highly porous structures of smooth and defect free nonwoven nanofibrous membranes. ${ }^{10}$ The purpose of this review is to highlight the application of emulsion electrospinning for the development of electrospun nanofibers to be used in both the food and pharmaceutical industries. The types of emulsion as well as the processing parameters are discussed in detail.

\section{Fabrication methods of electrospun nanofibers}

According to the method used for preparing polymers, electrospun nanofibers may be fabricated by two methods: (1) melt electrospinning, and (2) solution electrospinning. For stretching the jet of fluid, an electrical field is applied and after solidification, fibers are collected on the collector. Although similar principles are observed for these two methods, several obvious differences exist, such as the solidification mechanism and the resulting fiber diameter.

There are some advantages in the melt electrospinning application, compared to the solution electrospinning method: solvent-free process, high output due to no loss in mass by solvent evaporation, environmental friendly due to no recycling/ removal of toxic solvents, and ease of fabrication of polymeric fiber blends. ${ }^{11}$ However, some disadvantages of this method are the thermal degradation of polymers as a result of the high temperature and high viscosity of the polymer melt, and electric discharge problems due to poor conductivity. Polymer melt, with high viscosity and quick polymer solidification, performed by temperature gradient in the region between the needle tip and the collector, leads to difficulty in the submicron scale fiber fabrication; ${ }^{12}$ the temperature required for heating the polymer can be provided by heating oven, ${ }^{11}$ laser melting devices, ${ }^{13}$ or electric heating. ${ }^{14}$

In solution, electrospinning, solidification is carried out by fast solvent evaporation. However, some drawbacks of this method are related to the toxic solvents, environmental concerns and additional solvent extraction processes. To deal 
with the low productivity of solution electrospinning, enhancing the number of jets by adopting various techniques has led to the creation of different approaches, including multijets from single needle, multi-jets from multiple needles, and needleless systems. ${ }^{12,15}$

Another way to classify electrospinning is according to the manner of dispensation of the solution or melt, which is divided into two categories: (a) confined feed system (CFS), and (b) unconfined feed system (UFS). In the CFS, a constant rate is applied to inject the polymer solution or melt; however, the flow over the surface of another material in UFS is unconstrained. Application of CFS has the benefit of restricted flow rate, which results in better fiber quality and uniform fiber diameter, but it is susceptible to clogging. ${ }^{12,16}$ UFS, as reported by Thoppey et al. is an easily-implemented system, without the possibility of clogging and high potential for scale-up to fabricate high quality nanofibers. ${ }^{17}$

The type of solution and its properties (e.g. conductivity, viscosity, elasticity, and surface tension) could significantly influence the fabricated fiber characteristics and bioactive release profile. ${ }^{18}$ Some problems of traditional solutions, including severe initial burst release or formation of beaded fibers, require the need for using novel solutions. ${ }^{4}$ To overcome these limitations, emulsions are considered as a great alternative to produce relatively bead free fibers with sustained release.

\section{Emulsion-based delivery systems}

Emulsion-based systems are useful vehicles for encapsulating, protecting, and releasing valuable ingredients consisting of oil, surfactant/co-surfactant, and water. Conveniently, emulsion systems can be categorized based on their spatial organization of the oil and water phases into oil-in-water $(\mathrm{O} / \mathrm{W})$ and water-inoil (W/O) emulsions. In an $\mathrm{O} / \mathrm{W}$ emulsion, oil droplets are dispersed in the continuous water phase, while $\mathrm{W} / \mathrm{O}$ emulsions are dispersions of aqueous droplets in the oil phase. The substance that makes up the droplets in an emulsion is referred to as the "dispersed phase", while the surrounding liquid substance is called "continuous phase". ${ }^{19}$ Generally, the colloidal dispersions can be classified, based on their particle size, into conventional emulsions or macroemulsions, nanoemulsions, and microemulsions.

In conventional emulsions, the mean droplet sizes are in the range of $0.1 \mu \mathrm{m}$ to $100 \mu \mathrm{m}$, although it is possible to observe bigger and smaller particles in certain applications. Typically, for food-grade surfactants (e.g. phospholipids, proteins, polysaccharides), the thickness of the interfacial layer in conventional emulsions is between 1 to $10 \mathrm{~nm}$, but it might be thicker if biopolymer multilayers surround the particles. ${ }^{20}$ These systems are kinetically stable, but thermodynamically unstable and tend to break down over time as the result of different physicochemical mechanisms such as gravitational separation, flocculation, creaming, coalescence, and Ostwald ripening. Due to the simple structures and formulation, most conventional emulsion systems have only limited protection for active ingredients and it is difficult to control the release rate. The electrical charge on the particles can be controlled by using an appropriately charged surfactant, which can be positive, negative, or without charge. Conventional emulsions can be fabricated by homogenizing oil and aqueous phase together in the presence of a surfactant. Various homogenizers could be used for this purpose, using different homogenizing methods such as high-pressure homogenizer, ultrasonic homogenizer, and membrane homogenizer. ${ }^{19}$

A nanoemulsion is considered to be a conventional emulsion that contains very small particles $(100-200 \mathrm{~nm})$. Nanoemulsions tend to be clear or slightly turbid, due to the small lipid particle dimensions in comparison to the light wavelength, so the light scattering is relatively weak. ${ }^{21}$ Unlike conventional emulsions, which are mostly prone to gravitational separation and droplet aggregation, nanoemulsions are highly stable to gravitational separation because of their very tiny droplet size, which means that Brownian motion effects dominate the gravitational forces. ${ }^{22}$ In principle, a nanoemulsion could be prepared using oil and water without using an emulsifier. However, in practice, this system is highly unstable to droplet coalescence and needs a surfactant to simplify the formation of nanoemulsions and to improve its kinetic stability during storage. ${ }^{23}$ High-flow homogenization methods such as high-pressure microfluidic homogenization or ultrasonic emulsification are normally used in the formation of nanoemulsion systems. ${ }^{24,25}$ Application of the externally used shear and/or elongational flow could dominate the interfacial and internal viscous stress and will break down bigger particles into smaller particles. ${ }^{26}$ The advantage of very small particles of nanoemulsions is that any encapsulated compound could be diffused out of the carrier very quickly. Moreover, the very high ratio of surface area to volume in this system could accelerate different chemical reactions, which are taking place at the oil-water interface (e.g. lipid digestion). Thus, the bioavailability of the encapsulated substances within nanoemulsions is often much higher than that in conventional emulsion systems. ${ }^{27}$

A microemulsion is a thermodynamically stable, transparent, low viscous, and isotropic dispersion. This system could be prepared almost spontaneously by mixing oil, water, and surfactants together using low energy methods (e.g. vortexing, slow speed stirring) and contains very small particles (5-100 $\mathrm{nm})$. Compared to the conventional and nanoemulsion systems, microemulsions can be easily prepared, but they generally require higher concentrations of a surfactant alone, or in conjunction with a co-surfactant; ${ }^{28}$ i.e., nanoemulsions and microemulsions typically need fairly similar ingredients, but in different ratios, e.g., a higher surfactant-to oil ratio is needed to prepare a microemulsion than a nanoemulsion. ${ }^{23}$

Emulsion-based systems are widely used in both the food and pharmaceutical industries for the encapsulation, solubilization, entrapment, and controlled delivery of active ingredients. In the field of nanotechnology, these systems could also be applied to produce nanomaterials such as nanofibers (with a diameter of $100 \mathrm{~nm}$ or less) via electrospinning technology. ${ }^{29}$ Application of electrospinning using W/O emulsions could improve the release of hydrophilic encapsulated materials. ${ }^{30}$ For instance, for embedding enzymes, encapsulation through W/O emulsion electrospinning is considered to be a great alternative 
to keep enzymes from possible interactions with the external interface. ${ }^{31}$ In order to add lipophilic functional components to electrospun fibers, O/W emulsions can be utilized. For pharmaceutical applications, dissolution can be determined and modulated by the rate of dissolution of the carrier through dispersing drugs in carrier polymers using emulsion electrospinning. ${ }^{32}$

\section{Fabrication of emulsion-based electrospun nanofibers}

\subsection{Basic mechanism}

According to a wide range of research evaluating the electrospinning technique, there are four parts to the basic electrospinning setup, including a glass syringe containing a polymer solution, metallic needle, power supply, and metallic collector, ${ }^{29,33}$ as shown in Fig. 2. High voltage power is connected to the metallic needle and moves into the polymer solution, resulting in instability within the polymer solution, due to induction of charges on the polymer droplet. Simultaneously, a force that opposes the surface tension is generated by the reciprocal repulsion of charges and finally the polymer solution flows in the direction of the electric field. ${ }^{29}$ If the strength of the electrical field continues to increase, the deformation of the spherical droplet to a conical shape leads to the appearance of ultrafine nanofibers from the conical polymer droplet (Taylor cone). Fabricated nanofibers are collected from the metallic collector placed at a suitable distance. ${ }^{29,34}$

There are three different electrospinning techniques, including blend, coaxial and emulsion electrospinning, resulting in the incorporation of various active agents within or decoration on the outside of the nanofibers. Fig. 3 shows the cross-section of an individual fiber fabricated via the three methods in which blend electrospinning produces fibers containing the active agent dispersed throughout them, while fibers obtained by the other two methods have a core/shell morphology. ${ }^{35}$

In the blending electrospinning technique, bioactive molecules (e.g. drugs) are dissolved or dispersed (if insoluble) in the solution. Distribution of bioactive agent inside the fibers is highly dependent on the physicochemical properties of the solution and the interaction of the agent with solution. ${ }^{36}$ Although this technique is simple in comparison to coaxial and emulsion electrospinning, the application of this method has its own limitations. For instance, sensitive bioactive agents (e.g. proteins and cytokines) may be denatured in the presence of the solvents and lose their bioactivity. ${ }^{37}$ Moreover, regarding substance distribution, since most of the bioactive molecules are charged molecules, they will migrate into the jet surface as the result of charge repulsion during blending electrospinning. Thus, instead of a uniform distribution of the molecules, surface enrichment is generally observed in fibers (Fig. 2A). ${ }^{37}$

Coaxial electrospinning or co-electrospinning of core-shell micro- and nanofibers is a modification of the traditional electrospinning process consisting of two arranged nozzles, which are connected to a high voltage source. Two various solutions (core and shell materials) are pumped via nozzles, which results in a core-sheath fiber morphology (Fig. 2B). To avoid contact between solutions, both solutions remain separated until the last moment. In the coaxial electrospinning method, the biomolecule solution forms the inner jet, leading to more protection of the biomolecule, and is co-electrospun with a solution that forms the outer jet. ${ }^{38}$ The core and shell phase interaction have an important effect on the electrospinnability of the solutions and the best results are achieved by adding a common solvent to the two immiscible solvents of the core and sheath solutions. To avoid the jet break-up or getting fibers without a uniform core or sheath layer (fiber deposition), the ratio of the flow rates of the sheath and core solutions need to be adjusted to between $3: 1$ and $6: 1 .^{39}$ The coaxial electrospinning method can be used for the encapsulation of

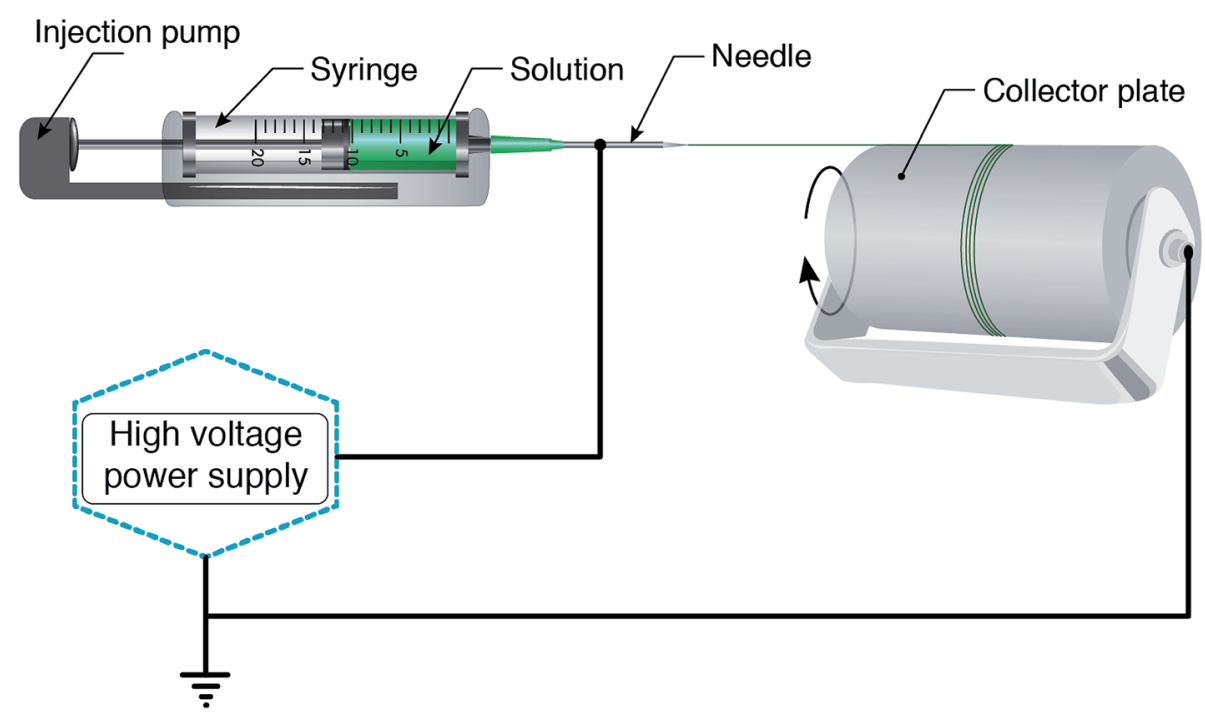

Fig. 2 Schematic of a typical setup for electrospinning. 


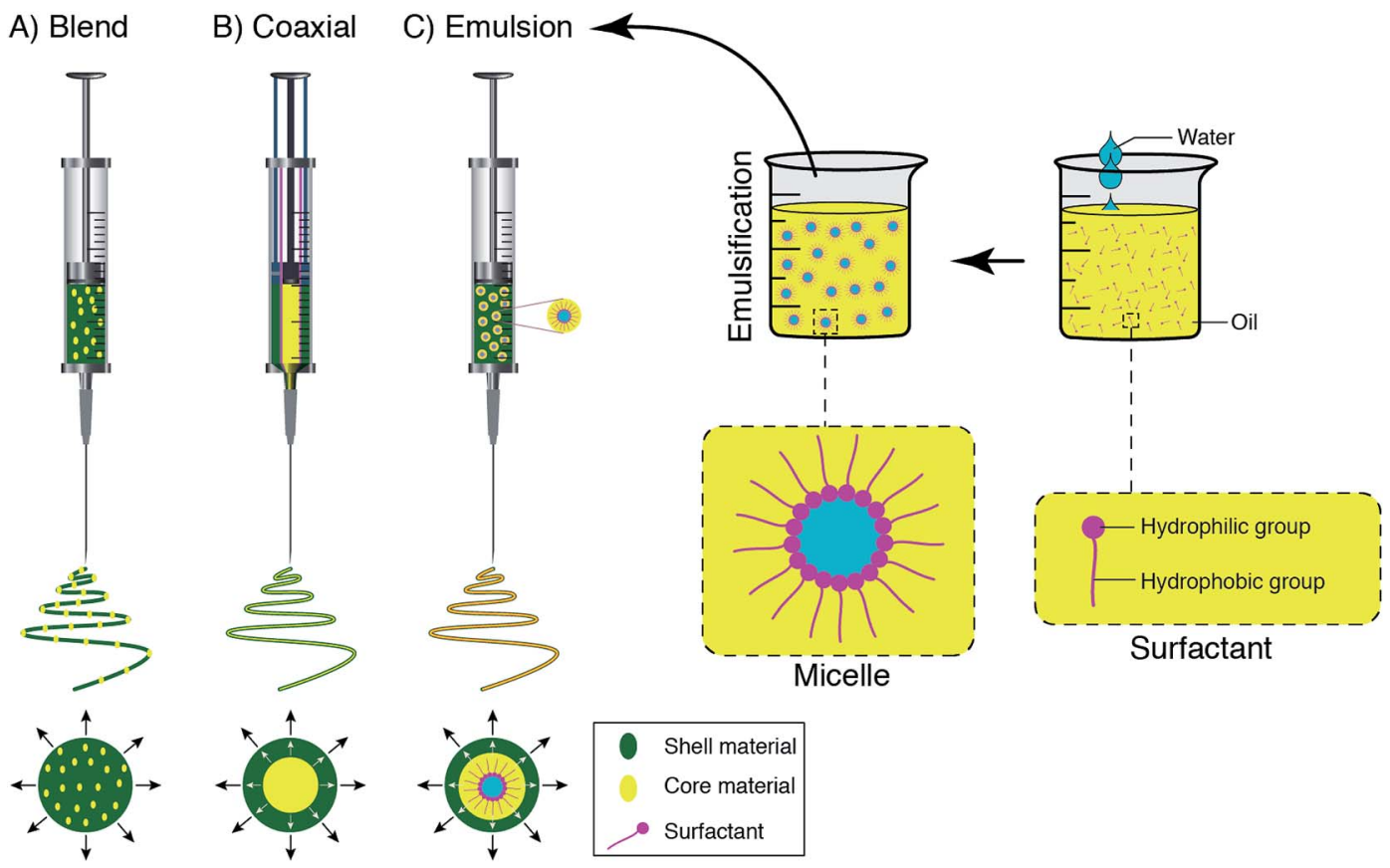

Fig. 3 Schematic displays of the spinneret loaded with a bioactive agent for (A) blend, (B) coaxial, and (C) emulsion electrospinning.

biologically active compounds, cell scaffolders and drug release, as well as the formation of multichannel nanotubes and nanofluidics. ${ }^{40}$ However, the application of this method also suffers from some disadvantages, including design complexity and the requirement of the precise control of process variables such as interfacial tension and viscoelasticity of the two polymers. ${ }^{41}$

The emulsion electrospinning method requires the same basic set up as blend electrospinning and involves the simultaneous spinning of two immiscible solutions (Fig. 2C). In this method, emulsification of active agents within the solution is carried out and they are dissolved in the appropriate solvents. In other words, the biodegradable fiber-forming polymer is solubilized in organic solvent to form the continuous phase (oil phase in case of using W/O emulsion), while the active agents are dissolved in aqueous solutions to form the water phase. Therefore, common solvents are eliminated, which is considered a main requirement of the blending technique. During electrospinning, the continuous phase rapidly evaporates, which results in an increase in the viscosity. Consequently, the aqueous phase droplets containing active ingredients migrate to the center of the jet as the result of the viscosity gradient. ${ }^{7}$ In the presence of the electric field, the droplets are unified due to the mutual dielectrophoresis that provides column-like structures and finally gives a fiber with a core-shell structure. ${ }^{9}$ Depending on the molecular weight of the bioactive molecules, they can be distributed within the fibers in terms of low molecular-weight application or form a core-shell fibrous structure (high molecular-weight)., ${ }^{7,42}$ Compared to coaxial electrospinning, this technique may still damage the bioactive components due to the interface tension between the aqueous and the organic phases of the emulsion. ${ }^{36}$ Both the basic electrospinning set-up and the process itself are relatively uncomplicated and there are several parameters that play a vital role in the successful outcome of the process. ${ }^{43}$

\subsection{Important electrospinning parameters and their effects}

There are different parameters that have great impact on the fabricated fiber properties; these parameters are categorized into three groups: process parameters, solution, and environmental parameters. ${ }^{29}$ Among the process parameters (e.g. applied voltage, solution flow rate and spinning distance), applied voltage is known to have a significant influence on nanofiber diameter, which varies from polymer to polymer. Sill and von Recum reported that the increase in the applied voltage resulted in the fabrication of nanofibers with smallerdiameters, which was related to the stretching of the polymer solution in correlation with the charge repulsion within the polymer jet..$^{34}$ In contrast, it was also demonstrated that there is a positive relation between nanofiber diameter and the applied voltage. Higher voltage results in the formation of beads or beaded nanofibers attributed to an increase in the jet length. ${ }^{44}$ Deitzel et al. also confirmed the formation of beaded nanofibers using poly(ethylene oxide) (PEO)/water by increasing the applied voltage. ${ }^{45}$ The solution properties determine the optimum applied voltage (e.g. conductivity, surface tension, and viscosity). ${ }^{46}$

The morphology of the electrospun nanofibers is affected by the flow rate of the solution and depends on the polymer system; flow rate adjustment results in nanofibers with uniform bead-free structures. ${ }^{33}$ A positive relation was observed by Megelski et al. between the nanofiber diameter of the electrospun polystyrene and the flow-rate of the polymer solution, attributed to the higher available volume of solution. ${ }^{47}$ Extreme 
flow rates lead to bead fiber formation associated with the remaining wet fibers before reaching the collector.

Changing the spinning distance can also affect the morphology of the nanofibers, where a greater distance between the metallic needle tip and collector may result in beaded nanofibers. ${ }^{48}$ Many studies have investigated the relation between the spinning distance and nanofiber diameter. It has been reported that nanofibers with smaller diameter will be produced by increasing the spinning distance, and vice versa. ${ }^{49,50}$

Concerning environmental parameters, temperature plays a critical role in nanofiber properties, due to its influence on the evaporation rate of the solvent and the solution viscosity. ${ }^{51}$ It was proven by De Vrieze et al. that thicker fibers were fabricated as a result of the higher viscosity caused by lower temperatures. ${ }^{52}$ Relative humidity (RH) is considered as another environmental parameter that highly depends on the chemical nature of the polymer. The role of humidity in determining the fiber diameter is attributed to its effect in controlling the solidification process of the charged jet. Park and Lee observed a reduction in the nanofiber diameter of polyethylene oxide (PEO) with increased humidity. ${ }^{53}$

\section{Applications of emulsion-based electrospun nanofibers}

\subsection{Food applications}

The food industry is an important field among a broad range of potential fields of application of electrospun nanofibers using emulsion electrospinning to encapsulate functional components. There are several bioactive compounds to be included in nanofibers, such as antimicrobial agents, enzymes, fatty acids and proteins (Table 1). To reduce microbial activities, the fabrication of electrospun nanofibers containing antibacterial and antifungal agents has garnered significant interest. Kriegel et al. incorporated eugenol (a lipophilic antimicrobial phytophenol, the predominant constituent of cloves (Syzygium aromaticum) essential oil) into a microemulsion of poly(vinyl alcohol) and cationic chitosan blended with a gemini surfactant (Surfynol 465). ${ }^{3}$ Investigation of the antimicrobial activity of fabricated nanofibers was carried out against two strains of Salmonella typhimurium and Listeria monocytogenes. The results indicated that the antimicrobial activity of the nanofibers (diameter range: 57 to $126 \mathrm{~nm}$ ) containing eugenol against Gram-negative bacterial strains was higher than Gram-positive bacteria. The pure eugenol microemulsion was found to have lower antimicrobial activity compared to eugenol nanofibers prepared with the emulsion electrospinning method, which is attributed to faster exhaustion and loss of antimicrobial activity in the free microemulsion. Moreover, a significant decrease in the average diameter was observed with higher surfactant concentration and lower eugenol concentration.

One major problem in developing enzyme applications in large-scale operations is their low catalytic efficiency and stability. To tackle this challenge, a few methods including genetic and protein engineering, ${ }^{68}$ solvent engineering, ${ }^{69}$ and enzyme entrapments in hollow fibers or microcapsules have been developed. ${ }^{70}$ In the enzyme immobilization method, the size of the carrier materials plays a key role. Greater size reduction results in higher efficiency of immobilized enzymes, due to the provision of higher enzyme loading per unit mass. Therefore, the use of the electrospinning method to produce nanofibers is considered an effective way to strengthen the functionality and the performance of enzymes. ${ }^{71}$ Dai et al. evaluated the activity of encapsulated laccase in microfibers prepared from the poly(DL-lactide) (PDLLA)/PEO-PPO-PEO (F108). ${ }^{5}$ They found that up to $67 \%$ of free enzyme activity remained after the electrospinning process. Moreover, they reported that by encapsulation of enzymes in microfibers, laccase could be applied in a wider range of $\mathrm{pH}$. In another study, lysozyme was encapsulated into core-sheath structured poly(DLlactide) fibers via emulsion electrospinning, and the release time reported was up to 2 weeks. ${ }^{41}$ In order to see the structure of fibers, laser confocal scanning microscopy was used and very porous and beadless fibers were observed. Other studies also confirmed the feasibility of using emulsion electrospinning to incorporate lysozyme into polycaprolactone (PCL) and a blend of polyethylene oxide (PEO) and PCL. ${ }^{72}$ According to the obtained data, a smaller amount of lysozyme was released from PCL fibers, in comparison to PEO/PCL fibers.

Among fatty acids, omega-3 polyunsaturated fatty acids, which have several health benefits, are considered as an important category that should be supplied through the diet. Different efficient strategies can be applied to protect these fatty acids from oxidation. Recently, García-Moreno et al. encapsulated fish oil (5, 7.5 and 10\% (w/w)) into poly(vinyl alcohol) (PVA) nanofibers, emulsified with whey protein isolate (WPI) or fish protein hydrolysate (FPH) via emulsion electrospinning. ${ }^{54}$ There was a positive correlation between fish oil load and average fiber diameter. This result is in agreement with the findings of Moomand and Lim, who reported $500 \mathrm{~nm}$ growth in fiber diameter as a result of an increase in the amount of fish oil $(30 \%(\mathrm{w} / \mathrm{w})) .{ }^{73}$ Considering oxidative stability, the peroxide value (PV) was evaluated and surprisingly, it was observed that unprotected fish oil had lower PV compared to nanofibers. This phenomenon may be attributed to the presence of trace quantities of metals (e.g. $\mathrm{Ca}, \mathrm{Fe}, \mathrm{Al}$ ) in PVA due to its production process in metal equipment. ${ }^{74}$ Moomand and Lim observed higher oxidative stability of zein nanofibers containing fish oil over a period of 14 days, due to the greater oxidation stability of zein, in comparison to PVA. ${ }^{73}$ However, the high cost of zein production makes it an uneconomical material for large-scale manufacturing.

Food compounds and environmental factors result in protein inactivation, in which these two factors limit the direct application of proteins in different food systems. Moreover, using traditional electrospinning for protein encapsulation also has some disadvantages (e.g. agglomeration and denaturation of proteins during mixing with polymer solutions, mostly accumulated on the surface of the fibers). ${ }^{75,76}$ To circumvent the drawbacks of traditional electrospinning, the feasibility of coreshell nanofibers fabricated via emulsion electrospinning based on L-limonene and hydrophobic polystyrene (PS) for protein 
Table 1 Application of the emulsion electrospinning technique for the fabrication of electrospun nanofibers ${ }^{a}$

\begin{tabular}{llll}
\hline & Emulsion \& polymer & $\begin{array}{l}\text { Electrospinning } \\
\text { parameters }\end{array}$ & Main results
\end{tabular}

\section{Food applications}

Eugenol

accase

Laccase

Fish oil

Bovine serum albumin

Lysozyme

Bovine serum albumin

Limonene
W/O emulsion: F108, PDLLA/methylene

dichloride solution

Microemulsion: Surfynol 465, water, glacial acetic acid, PVA

O/W emulsion: WPI, FPH, water, PVA, acetic acid

W/O emulsion: Span 80, PS, L-limonene, water

W/O emulsion: PBS, MC, PDLLA, chloroform

$\mathrm{W} / \mathrm{O}$ and $\mathrm{O} / \mathrm{W}$ emulsion: AOT, dichloromethane, alginate, water, calcium chloride solution, PLLA $\mathrm{O} / \mathrm{W}$ emulsion: formulation 1: PVA, limonene, water, formulation 2: PVA, water, Tween 20 , hexadecane
Glass syringe volume: $20 \mathrm{~mL}$, diameter capillary: $0.69 \mathrm{~mm}$, collector distance: $10 \mathrm{~cm}$, flow rate: 0.02 $\mathrm{mL} \mathrm{min}^{-1}$, voltage: 20 $\mathrm{kV}$, temperature: $25{ }^{\circ} \mathrm{C}$

Diameter capillary: 0.5 $\mathrm{mm}$, collector distance: $15 \mathrm{~cm}$, flow rate: $1.5 \mathrm{~mL}$ $\mathrm{h}^{-1}$, voltage: $12 \mathrm{kV}$, room temperature: 20 $\pm 2{ }^{\circ} \mathrm{C}$, humidity: $45 \%$

Collector distance: $10 \mathrm{~cm}$, flow rate: 0.02

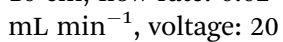
$\mathrm{kV}$, room temperature, collector plate size: $5 \times 5 \mathrm{~cm}$

Collector distance: $10 \mathrm{~cm}$, flow rate: $0.2 \mathrm{~mL}$ $\mathrm{h}^{-1}$, glass syringe volume: $3 \mathrm{~mL}$, voltage: $20 \mathrm{kV}$

Diameter capillary: $0.6 \mathrm{~mm}$

Diameter needle: 0.9 $\mathrm{mm}$, collector distance: 8-15 cm, voltage: $10-20 \mathrm{kV}$

Needle diameter: 0.8 $\mathrm{mm}$, collector distance: $20 \mathrm{~cm}$, flow rate: $5 \mu \mathrm{L}$ $\min ^{-1}$, voltage: $0.5-0.725 \mathrm{kV} \mathrm{cm}^{-1}$, temperature: $8-24{ }^{\circ} \mathrm{C}$, relative humidity: $55-85 \%$
- Higher antimicrobial activity of nanofibers containing eugenol, compared to pure eugenol, due to the slower release rate of eugenol from fibers

- Greater antibacterial effect against Gramnegative bacterial strains rather than Grampositives

- Positive correlation between the average diameter and eugenol concentration - Negative relation between the average diameter and surfactant concentration - The immobilized laccase activity was retained by over $67 \%$ of that of the free enzyme

- $50 \%$ of the initial immobilized laccase activity was maintained after 10 runs in the enzyme reactor

- Crystal violet dye was degraded by the prepared microfiber membranes - Immobilized laccase showed a wider pH range of catalysis activity

- Fibers fabricated from $10.5 \%$ (w/w) PVA-5\% $(\mathrm{w} / \mathrm{w})$ emulsion blend stabilized with WPI provided high omega-3 encapsulation efficiency $(92.4 \pm 2.3 \%)$ with an oil load capacity of $11.3 \pm 0.3 \%$

- Compared to emulsified and unprotected fish oil, the hydroperoxide contents and secondary oxidation products were higher in electrospun fibers

- The sustained release of protein from electrospun fibers was observed

- Higher PS molecular weight resulted in faster protein release rate and lower PS molecular weight caused more sustained release

- Evaporation rate of solvent had significant impact on protein dispersion

- Core-shell-structured ultrafine, porous and beadless fibers fabricated with efficient release time (up two weeks)

- The protein entrapment resulted in higher mass loss and greater reduction of the molecular weight of the matrix residues Longer release time of BSA $(120 \mathrm{~h})$ was obtained, compared to naked microspheres $(10 \mathrm{~h})$ attributed to the presence of Caalginate

- The effect of temperature was dependent on the PVA concentration of the emulsion - The relative humidity affected the morphology of the fiber and the fragrance encapsulation efficiency more than the temperature 
Table 1 (Contd.)

\begin{tabular}{ll}
\hline & \multicolumn{2}{l}{$\begin{array}{l}\text { Emulsion \& polymer } \\
\text { type }\end{array}$} \\
\hline Active compound & \\
Pharmaceutical applications \\
$\begin{array}{l}\text { Hydroxyapatite and } \\
\text { laminin }\end{array}$ & $\begin{array}{l}\text { W/O emulsion: PLCL, } \\
\text { HA, Span-80, } \\
\text { chloroform, laminin, }\end{array}$ \\
& $\begin{array}{l}\text { Tris buffered NaCl } \\
\text { W/O emulsion: PLGA, } \\
\text { DNA, DNA/chitosan }\end{array}$ \\
& DCM, HAp, water
\end{tabular}

Fluorescein isothiocyanatedextran (FITCdextran) Horseradish peroxidase

Human-nerve growth factor (NGF)

Doxorubicin hydrochloride

Levetiracetam

Cefradine and 5fluorouracil

Epidermal growth factor (EGF)
Electrospinning

parameters

Main results

Reference
Syringe diameter: 340 $\mathrm{mm}$, collector distance: $10 \mathrm{~cm}$, flow rate: $5 \mathrm{~mL}$ $\mathrm{h}^{-1}$, voltage: $10 \mathrm{kV}$

Collector distance: $15 \mathrm{~cm}$, flow rate: 0.012 $\mathrm{mL} \mathrm{min}^{-1}$, voltage: $17 \mathrm{kV}$ Collector distance: 15$20 \mathrm{~cm}$, flow rate: $0.4-0.6$ $\mathrm{mL} \mathrm{h}{ }^{-1}$, voltage: 14-16 kV

Syringe needle diameter: $0.9 \mathrm{~mm}$, collector distance: $15 \mathrm{~cm}$, flow rate: $1.0 \mathrm{~mL}$ $\mathrm{h}^{-1}$, voltage: $15 \mathrm{kV}$

W/O emulsion: PLGA, Span 80, chloroform, water, FITC

W/O emulsion: PLGA, chloroform/DMF, chitosan, acetic acid, PVA

Glass capillary tube inner diameter: $400 \mu \mathrm{m}$, voltage: $1.5 \mathrm{kV} \mathrm{cm}^{-1}$

Collector distance: $15 \mathrm{~cm}$, flow rate: 0.25 $\mathrm{mL} \mathrm{h}{ }^{-1}$, voltage: 14-16 kV

W/O emulsion: PEG750PLLA, PEG5000-PLLA, chloroform, SDS

W/O emulsion: PLGA, water, DCM, Tween 20

W/O emulsion: PLGA, chloroform, DMF, Span-80, water

W/O emulsion: PCL, chloroform, HA, Span 80, EGF, BSA

Collector distance: $18 \mathrm{~cm}$, flow rate: $50-70$ $\mu \mathrm{L} \min ^{-1}$, electric field strength: $2.5-2.8 \mathrm{kV}$ $\mathrm{cm}^{-1}$

$12 \mathrm{~cm}$, flow rate: $1.0 \mathrm{~mL}$ $\mathrm{h}^{-1}$, voltage: $18 \mathrm{kV}$,
Higher osteoblast proliferation and cell

4 maturation of PLCL/HA/laminin scaffolds were observed in comparison to PLCL/ laminin or PLCL/HA

- Incorporation of DNA/chitosan (added to the fabrication solution) proved to be the best way for DNA delivery

- HAp was effective for improving cell attachment for osteoblastic activity purpose and DNA release

The composite scaffold with sustained release of FITC-dextran (about 7 weeks) indicated great potential for bone regeneration

The distribution of horseradish peroxidase was discontinuous among the fibers; however, desirable encapsulation efficiency was obtained (up to $70 \%$ )

Emulsion electrospun fibers successfully encapsulated proteins and improved their release in a sustained manner

Changing the water phase in emulsions for electrospinning from water to PBS resulted in changing the water phase core from a continuous state to a discontinuous state in electrospun nanofibers

- The viability and proliferation in PLGA/ chitosan nanofibers was higher than PLGA fibers

- Optimum electrospinning was obtained at optimum concentration of PLGA and chitosan in the range of $12-16 \%$ and $4-6 \%$, respectively

- The release process of Dox was divided into two categories: (1) diffusion (66 wt\% in the first $50 \mathrm{~min}$ ), (2) enzymatic degradation (after $100 \mathrm{~min}$ )

- The released Dox showed the same antitumor activity against mice glioma cells as the original Dox

A nearly linear and constant release of levetiracetam from Pemulsion-coaxial electrospun fibers was reported over 20 days, while classical core-shell fibers had a linear release for 4 days followed by a steady state

- The emulsion electrospun fibers prepared with GE showed better hydrophilic and mechanical properties

- Fabricated electrospun nanofibers were less toxic and tended to improve the attachment of fibroblasts cells and proliferation

- EGF and HA were both encapsulated in nanofibrous scaffolds and simultaneously released

- The release of EGF and HA from nanofibers improved cell infiltration, up-regulated collagen and the TGF-b1 gene expression temperature $<25{ }^{\circ} \mathrm{C}$, air humidity: $<60 \%$ 
Table 1 (Contd.)

\begin{tabular}{llll}
\hline & Emulsion \& polymer & $\begin{array}{l}\text { Electrospinning } \\
\text { parameters }\end{array}$ & Main results \\
type & Reference
\end{tabular}
Metformin
hydrochloride or
metoprolol tartrate

\begin{abstract}
W/O emulsion: PCL, PHBV, Span 80,

chloroform, water
\end{abstract}
Flow rate: $1 \mathrm{~mL} \mathrm{~h}^{-1}$, voltage: $16 \mathrm{kV}$

\section{Rhodamine B and} bovine serum

albumin

W/O emulsion: CSTPVA, PCL, Span 80,

Rhodamine B
W/O emulsion: water, Span-80, PLGA, chloroform, DMF
Needle diameter: 0.6 $\mathrm{mm}$, collector distance: $13 \mathrm{~cm}$, flow rate: $1 \mathrm{~mL}$ $\mathrm{h}^{-1}$, voltage: $16 \mathrm{kV}$

Needle diameter: 1.0 inch, collector distance: 10 inches, voltage: 27 $\mathrm{kV}$ and enhanced the collagen III to collagen I ratio

- Epidermis regeneration was accelerated by the nanofibrous PCL/HA/EGF scaffold in the early phases of wound healing

- Application of the emulsion

electrospinning technique reduced the burst release and provided a sustained release of drugs, compared to blended electrospun nanofibers

- Compared to the PHBV, PCL showed a better drug delivery carrier and MPT incorporated nanofibers had less burst release

- Application of emulsion electrospinning methods resulted in reducing the initial drug burst release and provided a differential diffusion pathway to release - The presence of sodium citrate and various types of PVA resulted in the postponement of the maximum accumulated release of BSA The controllable release of Rhodamine B and excellent morphological sustainability were observed in a composite nanofiber mat, prepared by the emulsion electrospinning technique

\begin{abstract}
${ }^{a}$ PVA: poly(vinyl alcohol); F108: triblock copolymer PEO-PPO-PEO; PDLLA: poly(D,L-lactic acid); WPI: whey protein isolate; FPH: fish protein hydrolysate; PS: polystyrene; MC: methyl cellulose; PBS: phosphate buffered saline; BSA: bovine serum albumin; AOT: sodium bis(2-ethylhexyl) sulfosuccinate; PLLA: poly(L-lactic acid); PLCL: poly(L-lactic acid-co-e-caprolactone); HA: hydroxyapatite; PLGA: poly(lactide-co-glycolide); DCM: dichloromethane; HAp: hydroxylapatite; FITC: fluorescein isothiocyanate isomer I; FITC-dextran: fluorescein isothiocyanate-dextran; NGF: human-nerve growth factor; PLACL: poly(L-lactide- $c o$-e-caprolactone); PELCL: poly(ethylene glycol)- $b$-poly(L-lactide-co-caprolactone); DMF: $N, N$ dimethyl formamide; F127: Pluronic F127; CS-SH: thiolated chitosan; Span 80: sorbitan monooleate; HRP: horseradish peroxidase; PEGDA: poly(ethylene glycol)diacrylate; SDS: sodium dodecyl sulphate; PEG: methoxy-poly(ethylene glycol); Dox: doxorubicin hydrochloride; PCL: polycaprolactone; EGF: epidermal growth factor; PHBV: poly(3-hydroxybutyric acid-co-3-hydroxyvaleric acid); MPT: metoprolol tartrate; GE: gelatine.
\end{abstract}

encapsulation was evaluated. ${ }^{55}$ It was found that the higher molecular weight of PS polymer led to higher diameters of electrospun fibers. In addition, higher PS molecular weight, ranging between $280-350 \mathrm{kDa}$, resulted in a faster protein release rate and lower PS molecular weight $(75 \mathrm{kDa})$ and caused more sustained release. Another research effort confirmed this result, which reported a slower release rate of protein with lower molecular weight of the polymer. ${ }^{77}$ The protein release profile was divided into two steps, including initial burst release (during the first 2 days) and subsequent stable release (for more than 50 days). They demonstrated the key role of protein distribution within the fiber matrix in the release profiles and also indicated that the solvent evaporation rate has a significant impact on protein dispersion during fiber fabrication. Qi et al. demonstrated the good release behavior of bovine serum albumin (BSA) from poly(L-lactic acid) (PLLA) fibers prepared by emulsion electrospinning. ${ }^{56}$ They applied Ca-alginate as reservoirs, maintaining the full biological activity of BSA. This may be due to a mild gelation process resulting in the sustained release of BSA (for about $120 \mathrm{~h}$ ).

Emulsion electrospinning is considered to be an efficient method for protecting sensitive compounds against adverse conditions such as acidity and temperature. Limonene is a highly volatile and temperature-sensitive component. It was selected to be encapsulated in fibers fabricated by the electrospinning of emulsions of poly(vinyl alcohol) (PVA). ${ }^{57}$ In this study, the effects of two environmental parameters including temperature ( 8 to $24{ }^{\circ} \mathrm{C}$ ) and relative humidity ( 55 to $85 \%$ ) on the formation of fibers were investigated. Hexadecane, with low volatility and high melting point, was applied as the dispersed phase. Encapsulation efficiency, which is referred to as the ratio of actual to theoretical drug loading within the scaffolds, ${ }^{78}$ was measured by gas chromatography (GC). The highest encapsulation efficiency ( $67 \pm 6 \%$ ) was observed at the temperature of $16{ }^{\circ} \mathrm{C}$ and relative humidity of $55 \%$. Beaded fibers were produced at humidity higher than $55 \%$, attributed to the effect on the solvent evaporation rate and in fiber formation at different speeds. Zhang et al. also reported the influence of moisture on lowering the cohesive forces among polymer chains, which led to better limonene diffusion from the fibers based on ethylene vinyl alcohol copolymer. ${ }^{79}$ Emulsion containing hexadecane, in all conditions, resulted in bead fibers, which could be associated with higher viscosity of emulsion. 
To protect and also enhance the survival of probiotic bacteria and bacteriocins during their passage through the upper GI tract and during food processing and storage, electrospinning is of great interest due to the lack of severe conditions of temperature, pressure and chemicals required for sensitive compounds. ${ }^{80}$ Fung et al. investigated the feasibility of using soluble dietary fibers (SDF) from certain agricultural waste streams-okara (soybean solid waste), oil palm trunk (OPT), and oil palm frond (OPF) obtained via alkali treatment, for the encapsulation of Lactobacillus acidophilus using the electrospinning method. ${ }^{81}$ They found good bacterial survivability (78.6-90\%), as well as retained viability at refrigeration temperatures during the twenty one day storage study. In another attempt, the Bifidobacterium strains were encapsulated using a protein (whey protein concentrate (WPC)) and a carbohydrate (pullulan) as encapsulation material. ${ }^{82}$ Compared to pullulan, using WPC resulted in higher protection ability as it effectively prolonged the survival of the cells even at high relative humidity. The results revealed by Heunis et al. showed the potential of nanofibers prepared from various combinations of poly(D,L-lactide) (PDLLA) and poly(ethylene oxide) (PEO) for the encapsulation of bacteriocins (e.g. bacteriocin ST4SA produced by Enterococcus mundtii). ${ }^{83}$

\subsection{Pharmaceutical and biomedical applications}

Recently, the incorporation of electrospun nanofibers into a wide range of drugs has gained various levels of success for the treatment of different diseases (e.g. wound healing and cancer therapy). The electrospun nanofibers have also been applied in bone tissue engineering to enhance encapsulated bone mineral release. There are two main reasons causing bone degeneration, namely, age and disease (e.g. trauma and tumor removal). ${ }^{4}$ Among other solutions such as allografting, emulsion electrospinning is considered as a novel treatment method for proliferation, metabolism and maturation of human fetal osteoblasts.

Tian et al. incorporated hydroxyapatite (HA) and laminin within the shell and core of nanofibers, respectively, by emulsion electrospinning. ${ }^{4}$ Nanofibers were fabricated with different scaffolds, including poly(L-lactic acid-co-e-caprolactone)/hydroxyapatite (PLCL/HA), PLCL/laminin (PLCL/Lam) and PLCL/hydroxyapatite/ laminin (PLCL/HA/Lam). Results indicated that after a period of 21 days, PLCL/HA/Lam scaffolds had higher osteoblast proliferation compared to PLCL/Lam or PLCL/HA. Similar results were found for cell maturation on day 14 for PLCL/HA/Lam scaffolds. A synergistic function effect for both factors in the improvement of functionality of osteoblasts was observed.

In another study, incorporation of DNA into the scaffolds was carried out in three ways: (1) naked DNA, (2) DNA/chitosan nanoparticles incorporation into scaffolds after fiber fabrication by dripping, and (3) mixing DNA/chitosan nanoparticles with the poly(lactide-co-glycolide) (PLGA)/hydroxylapatite (HAp) solution before electrospinning. ${ }^{58}$ They demonstrated that as a result of the hydrophilic nature of HAp, faster DNA release occurred, and led to higher cell attachment. Hence, the potential for the use of the DNA/chitosan nanoparticle-encapsulated
PLGA/HAp composite scaffold (the third way) in bone tissue regeneration was reported. A fibrous scaffold prepared from PLGA/collagen incorporated with fluorescein isothiocyanatedextran (FITC-dextran) has been proven to indicate good osteoblastic activity. ${ }^{59}$ The authors reported the sustained release of FITC-dextran from composite fibers (with a mean diameter of $665 \mathrm{~nm}$ ) for about 7 weeks. They concluded that electrospun fibers fabricated by emulsion electrospinning have great potential for medical application, including bone regeneration.

A valuable treatment, which has a direct effect on the quality of human life, is nerve tissue repair or neuro-regeneration. In human tissue, extracellular matrix (ECM) is responsible for supporting and controlling living cells. Hence, bioactive protein encapsulation (i.e. nerve growth factor) in a polymeric scaffold with similar structure to ECM, such as electrospun fibers, could be an effective method for nerve tissue engineering. ${ }^{\mathbf{8 4 , 8 5}} \mathrm{Li}$ et al. incorporated human-nerve growth factor (NGF) into poly(L-lactide-co-caprolactone) fibers by emulsion electrospinning. ${ }^{6}$ The analysis of the bioactivity of NGF released from the fibers (diameter ranging from 600-900 nm) was determined by monitoring the differentiation of PC12 cells into neurons in the supernatant. The obtained data indicated that emulsion electrospun fibers can successfully encapsulate proteins and release them in a sustained manner.

There are other types of tissue regeneration, which benefit from electrospun fibers, including vascular and skin tissue reconstruction. Han et al. reported the good release behavior of horseradish peroxidase by fibers prepared from poly(L-lactideco-glycolide) (PLGA) encapsulated with chitosan hydrogel as a carrier. ${ }^{60}$ Results depicted that although the distribution of horseradish peroxidase was discontinuous, the encapsulation efficiency was up to $70 \%$, which could be considered as a suitable scaffold for vascular tissue engineering purposes. Ajalloueian et al. demonstrated that nanofibers fabricated from polylactic-co-glycolic acid (PLGA) and chitosan with emulsifier, namely polyvinyl alcohol (PVA) via emulsion electrospinning had good potential for application in skin tissue regeneration. ${ }^{\mathbf{6 1}}$ Optimum concentration ranges of PLGA and chitosan for the production of suitable mechanical fibers were $12-16 \%$ and $4-$ $6 \%$, respectively. Higher concentration of PLGA and chitosan resulted in increased viscosity of the emulsion.

There are several reasons why cancer therapy has extensively applied electrospun nanofibers for anticancer drug delivery. Some of these reasons include: reduction of toxic effects of anticancer drugs, greater possibility for selecting target organs, greater stability during blood circulation time and lower interactions with the reticuloendothelial system (RES). ${ }^{86}$ Doxorubicin hydrochloride (Dox) is a water-soluble anticancer drug incorporated into the ultrafine fibers consisting of a chloroform solution of amphiphilic poly(ethylene glycol)-poly(L-lactic acid) (PEG-PLLA) diblock copolymer. ${ }^{7}$ In vitro Dox release was evaluated by UV absorbance at $483.5 \mathrm{~nm}$ as a function of incubation time. Two release mechanisms of Dox were observed, including diffusion and enzymatic degradation. At the initial stages, diffusion was the major release mechanism (66 wt\% in the first $50 \mathrm{~min}$ ) and after $100 \mathrm{~min}$, enzymatic degradation was the main mechanism. By increasing proteinase $\mathrm{K}$ concentration, faster 
release was achieved. The antitumor activity of Dox-loaded PEGPLLA fibers was determined against mice glioma cells (C6 cell lines), in which fibers indicated relatively similar antitumor activity, in comparison to virgin Dox.

The incorporation of antibacterial agents in electrospun fibers for wound dressing is of particular interest. In vivo experiments demonstrated that scaffolds consisting of polycaprolactone (PCL), hyaluronan and encapsulating epidermal growth factor (EGF) accelerated the epidermis regeneration of wound healing (with a size of $18 \mathrm{~mm} \times 18 \mathrm{~mm}$ ) on the dorsum of rats. ${ }^{55}$ Hyaluronan, a glycosaminoglycan present in most organs of the human body, has a lubricating role and, by modulation of gene expression of some ECM proteins, plays a vital role in wound healing. ${ }^{87}$ The authors observed that hyaluronan, due to its high hydrophilicity, could enhance EGF release from the fibers. In another study carried out by Gomes et $a l .{ }^{88}$ the performance of three electrospun nanofiber mats, including a polyester (polycaprolactone, PCL), a protein (gelatin from cold water fish skin, GEL) and a polysaccharide (chitosan), regarding wound healing and cell-scaffold interaction, were compared. The highest impact on the healing process observed in in vivo tests, was found to be for chitosan, due to the reduction in wound contraction and improvement in production of the neodermis and re-epithelialization of the wound.

One major application field of electrospun fibers is drug delivery for different diseases including hypertension, high blood glucose and cholesterol. $\mathrm{Hu}$ et al. used emulsion electrospinning to fabricate nanofibers with poly(3-caprolactone) (PCL) or poly(3-hydroxybutyric acid-co-3-hydroxyvaleric acid) (PHBV) incorporated with either metformin hydrochloride $(\mathrm{MH})$ or metoprolol tartrate (MPT).$^{65}$ These two hydrophilic drugs are used to treat cardiovascular diseases. ${ }^{89}$ Differentiation in the physicochemical properties of PCL and PHBV led to significant variance in both release rate and drug distribution in fibers. Fibers fabricated by PHBV resulted in higher burst release, which may be attributed to the surface location of drugs in nanofibers as a result of the high crystallinity of PHBV (60$80 \%)$. On the other hand, the lower crystallinity (45-60\%) of PCL caused slower drug diffusion. It should be mentioned that significant differences were also observed among two drugs in terms of release profiles and their distribution. This can be associated with their molecular weights and other physicochemical characteristics such as hydrophilicity. In vitro cytotoxicity examination indicated no cytotoxicity effects of drugpolymer emulsion electrospun nanofibers, as well as a good biocompatibility of nanofibers with tissue cells. Authors reported a positive correlation between the number of live cells in all scaffolds and incubation time, with the highest live cell numbers of MPT-PCL.

Wang et al. developed a drug delivery system with the swelling core for the differential release of multiple drugs (e.g. Rhodamine B and bovine serum albumin (BSA)) using the emulsion electrospinning method. ${ }^{66}$ The core, prepared by the polyvinyl alcohol (PVA) aqueous solution, and the sheath composed of poly( $\varepsilon$-caprolactone) (PCL) dissolved in chloroform. Sodium citrate (SC) was also added, for the purpose of swelling regulation, in different ratios $(2 / 3,3 / 3,3 / 2$, and $3 / 4)$ with BSA. It was found that the ratio of SC to BSA had a notable impact on fiber morphology. To achieve smooth and uniform morphology, the optimum ratio of SC to BSA was determined to be $3 / 3$ and $3 / 2$. Higher concentrations of 40 and $30 \mathrm{mg} \mathrm{mL}^{-1}$ for BSA and SC, respectively, led to turbidity of the fibers. Another important factor affecting the morphology of the fibers was the change in the ratio of PVA to PCL, which changed the viscosity of the emulsion. A higher ratio of PVA to PCL caused higher viscosity, and resulted in the formation of ineligible fibers as well as lowered the bioactive release rate.

In another study, Rhodamine B was encapsulated within fibers prepared from poly(lactic-co-glycolic acid) (PLGA) and sorbitan monooleate (Span-80). ${ }^{67}$ It was concluded that the presence of Span-80 caused a rapid release of Rhodamine B and after the initial burst, prevented the quick diffusion of drug by lowering PLGA degradation. The Span-80 molecule has both hydrophilic and hydrophobic ends, leading to its surface location and delayed PLGA degradation. $\mathrm{Hu}$ et al. investigated the release efficiency of cefradine from poly(lactide-co-glycolide) (PLGA) prepared by emulsion electrospinning. ${ }^{63}$ The incorporation of protein gelatin (GE) into nanofibers was carried out in order to improve the surface properties for cell adhesion. During the first 24 hours, a burst release of cefradine was in the range of $40-50 \%$, and for the next 10 days a sustained release (about $80-90 \%$ ) was observed. This could be attributed to PLGA degradation during this period and drug diffusion..$^{90}$ The presence of GE caused a higher cefradine release rate, which might be due to the interaction of cefradine and GE, resulting in cefradine surface distribution and easier release. ${ }^{91}$

Viry et al. compared emulsion/coaxial electrospun fibers with coaxial electrospun fibers (prepared from PLGA) to evaluate their efficiency in the delivery of a highly soluble drug, namely, levetiracetam. ${ }^{62}$ According to the obtained release profiles, emulsion/coaxial fibers indicated a sustained release (about $47 \%$ ) over 18 days; however this release amount was observed after 4 days for coaxial fibers. This phenomenon may be attributed to a drug reservoir, which was in the whole core of the coaxial fibers, constituting small fragmented reservoirs in the core of emulsion/coaxial fibers, resulting in slower release due to the longer distance of diffusion.

For food, pharmaceutical, and biomedical applications, the fabrication of nanosized polymer structures in smallscale productions has been commonly presented. However, the development of electrospun products in large-scale industrial operations is still faced with several challenges such as the lack of the capability of properly managing the devolatilization of organic solvents and the efficient processability at relatively high throughput rates, the requirement of establishing an appropriate global legislation for nanosized electrospun materials, and the requirement of more human in vivo results to support already accomplished in vitro research and biomedical electrospun coatings development under laboratory conditions (e.g. following the guidelines of ISO 13485 for medical devices). ${ }^{\mathbf{2}}$ Moreover, mass production of nanofibers is another issue to overcome ${ }^{93}$ since a needle can produce only one polymer jet, needle electrospinning systems have very low productivity, 
typically less than $0.3 \mathrm{~g} \mathrm{~h}^{-1}$ per needle, making it unsuitable for practical applications. ${ }^{\mathbf{9 4}}$ However, as was mentioned, some techniques have been suggested to increase the productivity, such as systems with multiple needles. ${ }^{95}$

\section{Conclusions}

Emulsion electrospinning has been proven to have great potential for the encapsulation of both hydrophilic and hydrophobic bioactive compounds and drugs. Some problems of traditional solutions, including severe initial burst release or formation of beaded fibers, could be resolved using this method. The optimization of processing parameters plays a vital role in the successful encapsulation and release of active ingredients. The food and pharmaceutical industries are the two major fields that may benefit from electrospun nanofibers prepared via the emulsion electrospinning technique as the delivery system. The food industry utilizes electrospun nanofibers to encapsulate a wide range of active ingredients including proteins, antimicrobial agents and sensitive components. Some of the nanofiber applications in the pharmaceutical industry have been investigated in recent years, such as wound healing, tissue regeneration, disease treatment and drug delivery. However, further research needs to be conducted to evaluate the effect of different emulsion-based systems (e.g. nanoemulsions, multiple emulsions, multilayer emulsions, liposomal emulsions and niosomes, etc.) for fabrication of nanofibers using the electrospinning method. Although several studies have evaluated the applications of electrospun nanofibers produced through the emulsion electrospinning process in the pharmaceutical industry, no research has been conducted on the application of this system in food and agricultural industries (e.g. in food packaging, pesticides, etc.) that need to be investigated in the future.

\section{Acknowledgements}

Shahin Roohinejad would like to acknowledge the Alexander von Humboldt Foundation, Germany, for his postdoctoral research fellowship.

\section{References}

1 C. Wang, L. Wang and M. Wang, Mater. Lett., 2014, 124, 192196.

2 F. E. Ahmed, B. S. Lalia and R. Hashaikeh, Desalination, 2015, 356, 15-30.

3 C. Kriegel, K. M. Kit, D. J. McClements and J. Weiss, Langmuir, 2009, 25, 1154-1161.

4 L. Tian, M. P. Prabhakaran, X. Ding and S. Ramakrishna, J. Biomater. Sci., Polym. Ed., 2013, 24, 1952-1968.

5 Y. Dai, J. Niu, J. Liu, L. Yin and J. Xu, Bioresour. Technol., 2010, 101, 8942-8947.

6 X. Li, Y. Su, S. Liu, L. Tan, X. Mo and S. Ramakrishna, Colloids Surf., B, 2010, 75, 418-424.

7 X. Xu, L. Yang, X. Xu, X. Wang, X. Chen, Q. Liang, J. Zeng and X. Jing, J. Controlled Release, 2005, 108, 33-42.
8 A. Arecchi, S. Mannino and J. Weiss, J. Food Sci., 2010, 75, N80-N88.

9 J. C. Sy, A. S. Klemm and V. P. Shastri, Adv. Mater., 2009, 21, 1814-1819.

$10 \mathrm{Z}$. Li and C. Wang, in One-Dimensional nanostructures, Springer, Berlin Heidelberg, 2013, pp. 15-28.

11 H. Zhou, T. B. Green and Y. L. Joo, Polymer, 2006, 47, 74977505.

12 R. Nayak, R. Padhye, I. L. Kyratzis, Y. B. Truong and L. Arnold, Text. Res. J., 2012, 82, 129-147.

13 N. Ogata, S. Yamaguchi, N. Shimada, G. Lu, T. Iwata, K. Nakane and T. Ogihara, J. Appl. Polym. Sci., 2007, 104, 1640-1645.

14 J. Lyons, C. Li and F. Ko, Polymer, 2004, 45, 7597-7603.

15 A. L. Yarin and E. Zussman, Polymer, 2004, 45, 2977-2980.

16 Y. LIu and J.-H. He, Int. J. Nonlinear Sci. Numer. Simul., 2007, 8, 393-396.

17 N. M. Thoppey, J. R. Bochinski, L. I. Clarke and R. E. Gorga, Polymer, 2010, 51, 4928-4936.

18 D. I. Braghirolli, D. Steffens and P. Pranke, Drug Discovery Today, 2014, 19, 743-753.

19 D. J. McClements, E. A. Decker and J. Weiss, J. Food Sci., 2007, 72, R109-R124.

20 D. Guzey and D. J. McClements, J. Agric. Food Chem., 2007, 55, 475-485.

21 D. J. McClements, Adv. Colloid Interface Sci., 2002, 97, 63-89.

22 D. J. McClements and Y. Li, Adv. Colloid Interface Sci., 2010, 159, 213-228.

23 D. J. McClements, Soft Matter, 2012, 8, 1719-1729.

24 K. Meleson, S. Graves and T. G. Mason, Soft Mater., 2004, 2, 109-123.

25 T. S. H. Leong, T. J. Wooster, S. E. Kentish and M. Ashokkumar, Ultrason. Sonochem., 2009, 16, 721-727.

26 W. Lu, A. L. Kelly and S. Miao, Trends Food Sci. Technol., 2016, 47, 1-9.

27 E. Acosta, Curr. Opin. Colloid Interface Sci., 2009, 14, 3-15.

28 S. Roohinejad, I. Oey, J. Wen, S. J. Lee, D. W. Everett and D. J. Burritt, Food Chem., 2015, 174, 270-278.

29 A. Haider, S. Haider and I.-K. Kang, Arabian J. Chem., DOI: 10.1016/j.arabjc.2015.11.015.

30 H. Chen, J. Di, N. Wang, H. Dong, J. Wu, Y. Zhao, J. Yu and L. Jiang, Small, 2011, 7, 1779-1783.

31 C. Mateo, J. M. Palomo, G. Fernandez-Lorente, J. M. Guisan and R. Fernandez-Lafuente, Enzyme Microb. Technol., 2007, 40, 1451-1463.

32 Y. Yang, X. Li, W. Cui, S. Zhou, R. Tan and C. Wang, J. Biomed. Mater. Res., Part A, 2008, 86, 374-385.

33 V. Pillay, C. Dott, Y. E. Choonara, C. Tyagi, L. Tomar, P. Kumar, L. C. du Toit and V. M. K. Ndesendo, J. Nanomater., 2013, 2013, e789289.

34 T. J. Sill and H. A. von Recum, Biomaterials, 2008, 29, 19892006.

35 K. A. Rieger, N. P. Birch and J. D. Schiffman, J. Mater. Chem. $B, 2013,1,4531-4541$.

36 M. Zamani, M. P. Prabhakaran and S. Ramakrishna, Int. J. Nanomed., 2013, 8, 2997-3017. 
37 A. Szentivanyi, T. Chakradeo, H. Zernetsch and B. Glasmacher, Adv. Drug Delivery Rev., 2011, 63, 209-220.

38 I.-C. Liao, S. Chen, J. B. Liu and K. W. Leong, J. Controlled Release, 2009, 139, 48-55.

39 S. Chakraborty, I.-C. Liao, A. Adler and K. W. Leong, Adv. Drug Delivery Rev., 2009, 61, 1043-1054.

40 A. l. Yarin, Polym. Adv. Technol., 2011, 22, 310-317.

41 Y. Yang, X. Li, M. Qi, S. Zhou and J. Weng, Eur. J. Pharm. Biopharm., 2008, 69, 106-116.

42 Y. Yang, X. Li, L. Cheng, S. He, J. Zou, F. Chen and Z. Zhang, Acta Biomater., 2011, 7, 2533-2543.

43 J. Pelipenko, P. Kocbek and J. Kristl, Int. J. Pharm., 2015, 484, 57-74.

44 P. K. Baumgarten, J. Colloid Interface Sci., 1971, 36, 71-79.

45 J. M. Deitzel, J. Kleinmeyer, D. Harris and N. C. Beck Tan, Polymer, 2001, 42, 261-272.

46 A. Repanas, S. Andriopoulou and B. Glasmacher, J. Drug Delivery Sci. Technol., 2016, 31, 137-146.

47 S. Megelski, J. S. Stephens, D. B. Chase and J. F. Rabolt, Macromolecules, 2002, 35, 8456-8466.

48 X. Geng, O.-H. Kwon and J. Jang, Biomaterials, 2005, 26, 5427-5432.

49 K. P. Matabola and R. M. Moutloali, J. Mater. Sci., 2013, 48, 5475-5482.

50 T. Wang and S. Kumar, J. Appl. Polym. Sci., 2006, 102, 10231029.

51 Y. Su, B. Lu, Y. Xie, Z. Ma, L. Liu, H. Zhao, J. Zhang, H. Duan, H. Zhang, J. Li, Y. Xiong and E. Xie, Nanotechnology, 2011, 22, 285609.

52 S. De Vrieze, T. Van Camp, A. Nelvig, B. Hagström, P. Westbroek and K. De Clerck, J. Mater. Sci., 2009, 44, 1357.

53 J.-Y. Park and I.-H. Lee, J. Nanosci. Nanotechnol., 2010, 10, 3473-3477.

54 P. J. García-Moreno, K. Stephansen, J. van der Kruijs, A. Guadix, E. M. Guadix, I. S. Chronakis and C. Jacobsen, J. Food Eng., 2016, 183, 39-49.

55 X. Wang, Y. Yuan, X. Huang and T. Yue, J. Appl. Polym. Sci., 2015, 132, 41811.

56 H. Qi, P. Hu, J. Xu and A. Wang, Biomacromolecules, 2006, 7, 2327-2330.

57 A. Camerlo, A.-M. Bühlmann-Popa, C. Vebert-Nardin, R. M. Rossi and G. Fortunato, J. Mater. Sci., 2014, 49, 8154-8162.

58 H. Nie and C.-H. Wang, J. Controlled Release, 2007, 120, 111121.

59 K. Wei, Y. Li, H. Mugishima, A. Teramoto and K. Abe, Biotechnol. J., 2012, 7, 677-685.

60 F. Han, H. Zhang, J. Zhao, Y. Zhao and X. Yuan, J. Biomater. Sci., Polym. Ed., 2013, 24, 1244-1259.

61 F. Ajalloueian, H. Tavanai, J. Hilborn, O. Donzel-Gargand, K. Leifer, A. Wickham and A. Arpanaei, BioMed Res. Int., 2014, 2014, e475280.

62 L. Viry, S. E. Moulton, T. Romeo, C. Suhr, D. Mawad, M. Cook and G. G. Wallace, J. Mater. Chem., 2012, 22, 11347-11353.

63 J. Hu, J. Wei, W. Liu and Y. Chen, J. Biomater. Sci., Polym. Ed., 2013, 24, 972-985.
64 Z. Wang, Y. Qian, L. Li, L. Pan, L. W. Njunge, L. Dong and L. Yang, J. Biomater. Appl., 2016, 30, 686-698.

$65 \mathrm{~J} . \mathrm{Hu}, \mathrm{M}$. P. Prabhakaran, L. Tian, X. Ding and S. Ramakrishna, RSC Adv., 2015, 5, 100256-100267.

66 Y. Wang, Z. Li, P. Shao, S. Hao, W. Wang, Q. Yang and B. Wang, Mater. Sci. Eng., C, 2014, 44, 109-116.

67 Y. Liao, L. Zhang, Y. Gao, Z.-T. Zhu and H. Fong, Polymer, 2008, 49, 5294-5299.

68 U. T. Bornscheuer and M. Pohl, Curr. Opin. Chem. Biol., 2001, 5, 137-143.

69 A. M. Klibanov, Nature, 2001, 409, 241-246.

70 Z. Ujang, N. Al-Sharbati and A. M. Vaidya, Biotechnol. Prog., 1997, 13, 39-42.

71 H. Jia, G. Zhu, B. Vugrinovich, W. Kataphinan, D. H. Reneker and P. Wang, Biotechnol. Prog., 2002, 18, 1027-1032.

72 T. Briggs and T. L. Arinzeh, J. Biomed. Mater. Res., Part A, 2014, 102, 674-684.

73 K. Moomand and L.-T. Lim, Food Res. Int., 2014, 62, 523-532. 74 X. Zhu, Q. Gao, D. Xu and X. Shi, J. Appl. Polym. Sci., 2009, 113, 143-149.

75 X. Huang and C. S. Brazel, J. Controlled Release, 2001, 73, 121-136.

76 Y. K. Luu, K. Kim, B. S. Hsiao, B. Chu and M. Hadjiargyrou, J. Controlled Release, 2003, 89, 341-353.

77 F. Boury, H. Marchais, J. P. Benoit and J. E. Proust, Biomaterials, 1997, 18, 125-136.

78 X. Zhao, Y. S. Lui, P. W. J. Toh and S. C. J. Loo, Materials, 2014, 7, 7398-7408.

79 Z. Zhang, L.-T. Lim and M. A. Tung, J. Appl. Polym. Sci., 2001, 79, 1949-1957.

80 B. Ghorani and N. Tucker, Food Hydrocolloids, 2015, 51, 227240.

81 W.-Y. Fung, K.-H. Yuen and M.-T. Liong, J. Agric. Food Chem., 2011, 59, 8140-8147.

82 A. López-Rubio, E. Sanchez, S. Wilkanowicz, Y. Sanz and J. M. Lagaron, Food Hydrocolloids, 2012, 28, 159-167.

83 T. Heunis, O. Bshena, B. Klumperman and L. Dicks, Int. J. Mol. Sci., 2011, 12, 2158-2173.

84 N. Ashammakhi, A. Ndreu, A. Piras, L. Nikkola, T. Sindelar, H. Ylikauppila, A. Harlin, E. Chiellini, V. Hasirci and H. Redl, J. Nanosci. Nanotechnol., 2006, 6, 2693-2711.

85 N. Bhattarai, D. Edmondson, O. Veiseh, F. A. Matsen and M. Zhang, Biomaterials, 2005, 26, 6176-6184.

86 L. Liu, C. Li, X. Li, Z. Yuan, Y. An and B. He, J. Appl. Polym. Sci., 2001, 80, 1976-1982.

87 L. Zhao, H.-J. Gwon, Y.-M. Lim, Y.-C. Nho and S. Y. Kim, Carbohydr. Polym., 2014, 102, 598-605.

88 S. R. Gomes, G. Rodrigues, G. G. Martins, M. A. Roberto, M. Mafra, C. M. R. Henriques and J. C. Silva, Mater. Sci. Eng., C, 2015, 46, 348-358.

89 W. L. Lee, P. Wee, C. Nugraha and S. C. J. Loo, J. Mater. Chem. $B, 2013$, 1, 1090-1095.

90 S. Yan, L. Xiaoqiang, L. Shuiping, M. Xiumei and S. Ramakrishna, Colloids Surf., B, 2009, 73, 376-381.

91 H. Nie, J. Li, A. He, S. Xu, Q. Jiang and C. C. Han, Biomacromolecules, 2010, 11, 2190-2194. 
92 S. Torres-Giner, R. Pérez-Masiá and J. M. Lagaron, Polym. Eng. Sci., 2016, 56, 500-527.

93 Y. Wan, J. Qiang, L. Yang, Q. Cao and M. Z. Wang, Adv. Mater. Res., 2014, 843, 9-13.
94 H. Niu, T. Lin and X. Wang, J. Appl. Polym. Sci., 2009, 114, 3524-3530.

95 Y. Echegoyen, M. J. Fabra, J. L. Castro-Mayorga, A. Cherpinski and J. M. Lagaron, Trends Food Sci. Technol., 2017, 60, 71-79. 